\title{
Multi-Domain Network Slicing With Latency Equalization
}

\author{
Ivana Kovacevic, Student Member, Alireza Shams Shafigh, Member, Savo Glisic Senior Member, Beatriz \\ Lorenzo, Member, and Ekram Hossain Fellow, IEEE
}

\begin{abstract}
With network slicing, physical networks are partitioned into multiple virtual networks tailored to serve different types of service with their specific requirements. In order to optimize the utilization of network resources for delay-critical applications, we propose a new multi-domain network virtualization framework based on a novel multipath multihop delay model. This framework encompasses a novel hierarchical orchestration mechanism for mapping network slices onto physical resources and a mechanism for dynamic slice resizing. The main idea is to locally redefine the delay requirements on each network domain depending on the conditions in the rest of the network. Delays larger than threshold (debt) are allowed in certain domains if there is a possibility to compensate such excessive delays in other segments of the network that can transmit the messages with less latency (credit). This tradeoff or delay threshold redefinition on different segments of the route is referred to as network latency equalization. For performance comparison, minimum cost routing with latency constraints is used as a baseline. We show that our approach enables significantly better utilization of the network resources measured in the number of slices with the same latency requirements that can be accommodated in the network.
\end{abstract}

Index Terms - Real-time applications, multi-domain network slicing, end-to-end network slicing, resource allocation, end-toend latency, limited-latency networks.

\section{INTRODUCTION}

Network slicing was initially proposed in the context of $5 \mathrm{G}$ [1], [2] as one of the key technologies allowing diverse services and applications to coexist on the common infrastructure. ITU-T defines network slice (NS) as a logical network that provides specific network capabilities and network characteristics to its tenant [3]. It enables network operators to create service-aware logical networks customized for different vertical industries, which have diverse requirements in terms of functionality, performance and resource separation. An NS is composed of different physical or virtual network elements, resources and functions including networking, storage and computing [1]. By sharing the same infrastructure, expenses of

The work of I. Kovacevic, A. S. Shafigh and S. Glisic was supported by the Finnish Academy/NSF US collaborative program WiFiUS 2018. The work by E. Hossain was supported by a Discovery Grant from the Natural Sciences and Engineering Research Council of Canada (NSERC).

I. Kovacevic, A. S. Shafigh and S. Glisic are with the Centre for Wireless Communications, University of Oulu, 90570 Oulu, Finland (email: firstname.lastname@oulu.fi). B. Lorenzo is with the Dept. Electrical and Computer Engineering at University of Massachusetts, Amherst, USA (email: blorenzo@umass.edu), and formerly with the University of Vigo, 36310 Vigo, Spain. E. Hossain is with Department of Electrical and Computer Engineering at University of Manitoba, Winnipeg, Canada (email: Ekram.Hossain@umanitoba.ca). network expansion and operation can be significantly reduced as reported by Liang et al. in [4], and Chowdhury et al. in [5].

New use cases such as delay-critical applications require End-to-End (E2E) Quality of Service (QoS) guarantees provided over increasingly complex heterogeneous infrastructure. Several characteristics of future networks should be considered when providing E2E service as suggested by Bernardos and et al. in [6]. First, different network technologies often belong to different network domains that are autonomously managed. This is not only the case between different operators but can occur inside the network of the same operator. Second, operators cover certain geographical regions, and they have to cooperate with others in order to provide certain services. For example, one high definition video live stream E2E route may span over multiple wireless access, local area and core network domains. Finally, many services require multiple different specialized providers (e.g. mobile operator, cloud service provider, transport network provider). For these reasons, recently, research efforts have been made toward extending the 5G slicing concept to include multi-provider and multidomain aspects. Referent frameworks are proposed by IETF and summarized by Bernardos and et al. in [6] currently in the form of internet draft, ETSI group report summarized by Makhijani et al. in [7] and IRTF summarized by Galis et al. in [8]. One of the main characteristics of multi-domain slicing is that interconnected domains are autonomously managed, and E2E slice can be seen as a concatenated network of subnetworks belonging to different network domains. In this paper, by domain we refer to an autonomous part of the E2E communication network owned and controlled by a network operator. During the slicing process, it is not possible for a single entity performing slicing to be aware of the topology and resource availability across the world wide network. Also, it is not desirable for a domain or subnetwork to share its internal topology with other domains and entities [7]. For lack of a centralized entity with knowledge of the whole network that could calculate a global optimal solution, multi-domain slicing has to be approached in a decentralized manner [7] [9] [10]. Therefore, ETSI proposes a hierarchical referent architecture for slice orchestration [7], where no entity is required to have global knowledge of the network. More details are presented in Section III A. Within this framework, several research challenges remain to be studied. One of the important challenges that we address in this paper is how to efficiently allocate physical resources to network slices to guarantee a certain E2E delay for limited latency traffic.

Recently multiple potential low latency applications, such 
as high-capacity cloud services, mission-critical machinetype communication, tactile internet and high-resolution video streaming services -virtual reality (VR), augmented reality (AR)- have been discussed. These applications have different requirements in terms of data throughput, latency and reliability, and the need for further classification of low latency traffic emerged. QoS requirements for different low latency use cases can be found in [11] reported by Jiang et al. and [12]. Furthermore, should be noted that even the definition of communication latency itself is not unique, since it depends on the use cases [11]. Latency requirements can be expressed by stochastic measures, such as expected value and the variance of the latency must remain under a predefined threshold as defined by Weiner et al. in [13], and Yamamoto et al. in [14], or that latency should be under a certain threshold with defined probability. In the literature, the former case is also referred to as probabilistic latency while the later as deterministic latency as defined by Nasrallah et al. in [15]. In this paper, we consider the second latency definition and focus on the special type of traffic where delay threshold is not defined per packet but per group of packets that form a frame or a message. This definition is appropriate for use cases such as VR, AR, and real-time control, and we will refer to this type of traffic as message-latency-limited traffic.

In this paper, we propose a novel decentralized dynamic end-to-end network slicing (DEENS) framework for messagelatency-limited traffic over multi-domain networks. The algorithm is designed to minimize usage of the resources, which enables maximizing the number of slices the network can accommodate while keeping the latency strictly under the required threshold.

In summary, our paper presents the following major contributions:

- An adaptive three-phase multi-domain slicing algorithm, for mapping of network slices to physical network resources for limited-latency services with minimum utilization of the network resources. The multi-domain network model, considered in this paper, is motivated by the fact that the Internet is a multi-domain architecture, and there is a growing interest to model and optimize $6 \mathrm{G}$ as a network of subnetworks [10],

- Analysis of capacity requirements to achieve delay for a special type of traffic characterized by high throughput and limited latency per message such as VR traffic,

- A mechanism for augmentation/resizing of NSs during operation time by horizontal and vertical latency equalization. Latency equalization is used to: a) increase the number of accepted slices in the initial slice formation process and b) handle the variation of the required resources due to variation of the traffic during the slice life time (slice operation time).

The rest of the paper is organized as follows: Section II presents related works on network slicing. Section III provides the general system model. In Section IV we characterize E2E latency and reliability. Our proposed dynamic slicing algorithm is developed in Section V, while the credit and bonus trading algorithm for slice equalization is presented in Section
VI. Section VIII provides numerical results illustrating the performance of our schemes. Finally, Section IX concludes the paper. A list of notations used throughout the paper is given in Table I.

\section{RELATED WORK}

In a recent work Afolabi et al. [9] propose a softwaredefined network (SDN) architecture for network slicing across multiple administrative domains. The authors conclude that network slicing should be orchestrated in an hierarchical fashion. In our work, we develop this idea and extensively study the problem of resource allocation and establishment of a network slice over the multiple network domains for message latency limited traffic. Note that SDN does not provide a solution for the slicing problem itself, such as how and when slices should be formed, but rather enables the implementation of slicing schemes. An SDN framework enables flexibility, programmability, and simpler management for the network. It does so by decoupling the data from control plane as in [16] by Kreutz et al. and [17] by Hu et al. The control plane is centralized, which allows handling of complex tasks, such as deployment and management of wireless network slices. Examples of SDN designs for the wireless domain can be found in [18], [19] proposed by Yang et al, Pentikousis et al. and Qadir et al. respectively. The concept of hierarchical multidomain slicing architecture is defined by Makhijani et al. in ETSI report [7]. We will discuss details of this framework in next section. In another ETSI document by Bernardos et al. [6] several similar hierarchical frameworks are discussed for multi-domain network virtualization with focus on Network Function Virtualization (NFV) placement.

Many works on slicing deal with joint resource allocation and Virtual Network Function (VNF) placement as reported by Guan et al. in [20] Wang et al. in [21] and Leconte et al. in [22]. In this work, we address slice tracing and focus on the resource request problem that is specific to low latency traffic in a multi-domain scenario. VNF placement inside a subnetwork of a multi-domain scenario does not differ from placement inside a single domain scenario and, therefore, is not considered in this work.

The problem of low-latency for critical traffic in a single domain is considered by Kumar et al. [23]. The authors propose a heuristic algorithm for finding constrained paths in an SDN network. A mapping algorithm based on linear programming is proposed by Lu et al. [24] as a solution to the problem of low-latency network slice establishment. In this work, the complexity of the solution is reduced by simplifying the latency parameters and dividing the large-scale slices into several subgraphs. While the slices are formed with the objective of minimizing overall latency in the slice, the model does not consider guaranteeing the E2E delay that a particular user is experiencing. Sun et al. in [25] propose a resource allocation policy that maximizes energy efficiency in an ultra-reliable low-latency network. This work is limited to a wireless access network. Other examples of resource allocation frameworks for access networks include work by She et al. [26], which considers low latency requirements in vehicular 
communications systems, and work by Rahman et al. [27], which studies ultra-low latency in fog radio access networks. The resource allocation in the entire network is studied as a multi-period network utility maximization (NUM) problem with the constraints on the average E2E delay. Although the authors propose an optimal distributed delay-aware dynamic network utility maximization algorithm as a solution to this problem, the average delay constraint has limited application for future low-latency services.

Several works propose minimum cost routing with E2E delay constraints for low latency traffic as reported by Fendt et al. in [28], Hajiesmaili et al. in [29] and Kumar et al. in [23]. Overall, our work is different in two ways. First, in our case the delay requirement per domain depends on the availability of the resources in the rest of the network as well. The delay thresholds for different domains are a result of interdomain negotiation, so it is necessary to initially find paths with low delay even if they do not satisfy the nominal delay constraint. For some of these paths, the inter-domain delay equalization may bring the delay below the required threshold. The second difference is that our objective is to accommodate as many NSs as possible for the given network resources. This objective represents a trade-off between minimizing cost and maximizing number of customers, and it is essentially different from minimum cost routing. We employ a version of the minimum cost routing algorithm only for initial interdomain path computation and use latency equalization to further increase the number of accepted slice requests.

\section{System MODEL AND ASSUMPTIONS}

\section{A. Relation to ETSI Definitions and Terminology}

In this paper, we use the following terminologies defined by ETSI [7]. Network slice is a description of a serviceaware logical network that is composed of different physical or virtual network elements, resources and functions. A complete network slice is an inter-connection of subnetwork (domain) slices. The user of the network slice is called a 'tenant' and the tenant defines service requirements. Network Slice Instance (NSI) is an instance of a type of network slice that has resources allocated to it from underlying network infrastructure and is independently managed and monitored by the tenant. The multi-domain slicing referent architecture has three main components: Tenant, Network Slice Provider (NSP) and Network Slice Agent (NSA). NSP is an entity that provides access to a network slice instance and the resources associated with it. An NSP communicates with multiple NSAs that are network slice entities in an infrastructure provider's domain. An NSP does not have knowledge of each domain topology but is aware of how domains are interconnected. An NSA is capable of extracting the topology and operational state of its own network and thereby coordinating with the NSP to maintain its own portion of the network slice. The operation to instantiate a network slice on the infrastructure consists of path computation (tracing) and mapping of a network slice on the infrastructure. The NS mapping function is a mechanism to create associations between infrastructure elements and logical elements in network slice instances. The resource computation function determines the suitable paths (traces) and necessary resources for NS implementation. It is performed by NSP in cooperation with NSAs since domains are independently managed. In this paper, we elaborate on the resource computation function for the message-latency-limited traffic case while for the other network slice functions in a multi-domain scenario, we refer the readers to [7]. In addition to the functionality envisioned by the ETSI framework, our resource computation function focuses on maximization of the resource utilization to accommodate the maximum number of accepted slice requests. The network slicing requires a dynamic computation. Therefore, resource availability databases are updated based on discovery function and memorizing available and allocated resource information.

\section{B. Network Model}

We consider a heterogeneous network, an example of which is shown in Fig. 1, with $M$ domains operated by different network operators (NOs) represented by the set $\mathcal{M}=$ $\{1,2, \ldots, M\}$. These domains provide all required physical resources (e.g. network devices, sub-channels, power, wired and wireless links) to $K$ service providers (SPs) denoted by $\mathcal{K}=\{1,2, \ldots, K\}$.

Each NO $m$ possesses and operates a physical network represented by a physical weighted graph $G^{m}=\left(\mathcal{I}^{m}, \mathcal{L}^{m}, \mathcal{C}^{m}\right)$, where $\mathcal{I}^{m}$ and $\mathcal{L}^{m}$ denote the sets of physical nodes and links of NO $m$, respectively. $L^{m}$ is the number of links and $I^{m}$ is the number of nodes in domain $m$. The capacities of links in $\mathcal{L}^{m}$ are given in the vector $\mathcal{C}^{m}=\left[C^{m, 1}, \ldots, C^{m, l}, \ldots, C^{m, L^{m}}\right]$, where each element $C^{m, l} \geq 0$ is the capacity of link $l^{m} \in \mathcal{L}^{m}$.

An SP $k$ has a set of message-latency-limited users $\mathcal{U}_{k}$, that are randomly scattered throughout the network. An SP requests a network slice in order to serve a number of sessions for these users. This is a long-term partitioning of the network, and we assume that it is performed before the service starts. Once the slices are instantiated, the network is able to guarantee the latency previously agreed in the contract with SP. Session $s$ is established between source $U_{k, s}$ and its destination $U_{k, s}^{\prime}$, where $U_{k, s}, U_{k, s}^{\prime} \in \mathcal{U}_{k}, s \in \mathcal{S}_{k}$ and $\mathcal{S}_{k}$ is the set of all sessions of SP $k$.

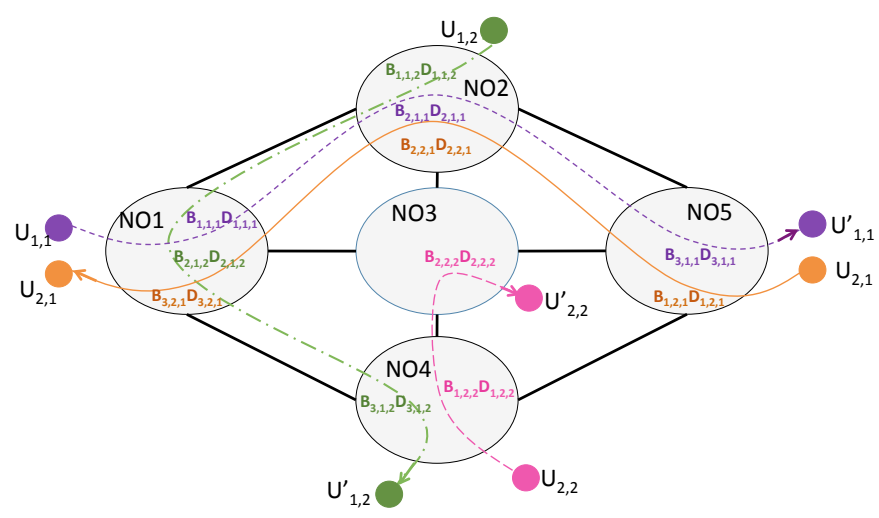

Fig. 1. Multi-domain network. 


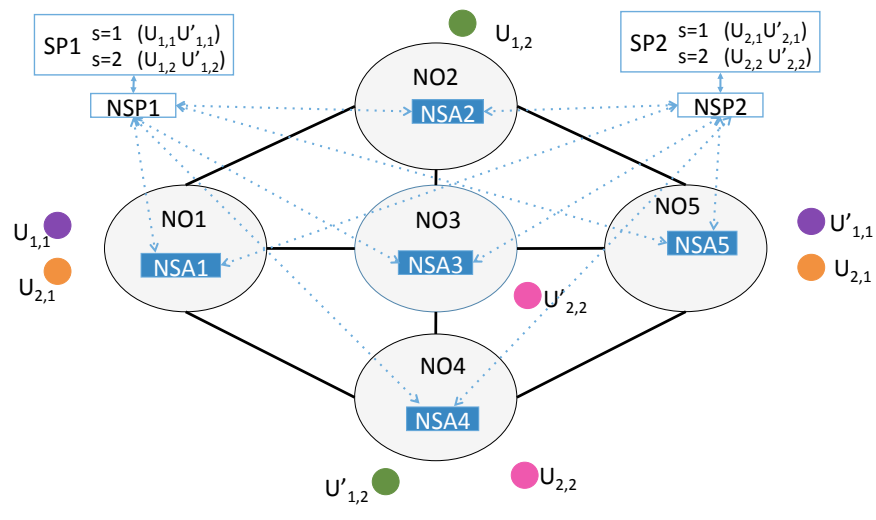

Fig. 2. Referent component architecture.

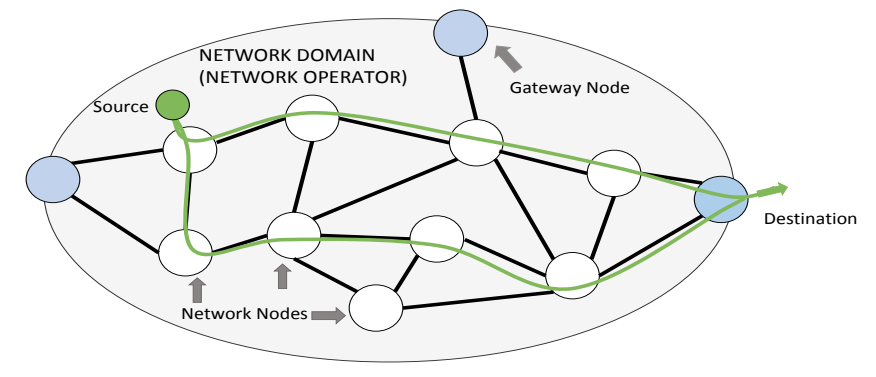

Fig. 3. Domain model.

\section{Latency-Limited Traffic Resource Request in a Multi- Domain Network}

In contrast to the single-domain scenario where latency constraint can be easily incorporated into an NS request, a multi-domain scenario faces unique challenges. Due to the lack of an entity with knowledge of the global topology and resource availability [7], a domain slice request has to be passed to each domain on the inter-domain path. Since E2E delay is the sum of delays through all domains with different resource availability on the path, it is not straightforward what should be the latency constraint requested from each domain. Our proposed slicing algorithm provides a solution where delay requirements can be negotiated among the domains in a cooperative way without compromising domains' autonomy and privacy.

\section{Reference Framework for Multi-Domain Network Slicing}

The ETSI generalized architecture allows different network service providers to coordinate and concurrently operate different services by using active network slices. A tenant, which is a limited-latency SP in our model, uses a network slice to accommodate multiple communication sessions. An NSP provides a network slice as a service to the tenant. Each tenant has its own NSP. Each domain has its NSA that knows the domain's topology and maps NSP requests within its domain. The NSA coordinates with multiple NSPs. The referent component architecture for the scenario in Fig. 1 is presented in Fig. 2.

SP $k$ requests a network slice to serve its $\mathcal{S}_{k}$ sessions. Each NS is a collection of sub-NS (SNS) dedicated to individual sessions. Each SNS can be further decomposed into the domain SNS (DSNS).

In the next subsections, we will describe the slice tracing process to find the potential paths, i.e. network infrastructure (nodes and links) to which a network slice can be mapped. Tracing is performed for each session individually.

\section{E. Inter-Domain Slice Tracing}

The inter-domain network topology of the entire network can be presented as a graph where vertices represent network domains and edges represent connections among them. The topology matrix $\mathbf{W}_{M \times M}$ has entries $w_{m, m^{\prime}}$ equal to the weight of inter-domain connection that reflect the general resource availability $\bar{C}^{m \prime}$ in the domain $m^{\prime}$ for the extension of the slice from domain $m$. One option to obtain general resource availability is to find total capacity of all links in the domain and scale it with the size of network, i.e. number of links. We define $\bar{C}^{m}=\left(\sum_{l \in \mathcal{L}^{m}} C^{m, l}\right) / L^{m}$. The interdomain link weight for the purpose of shortest path routing algorithm should be defined as $w_{m, m^{\prime}}=1 / \bar{C}^{m^{\prime}}$. The NSP of each SP has knowledge of the topology matrix W. An NSP receives updates about general resource availability from the NSA of each domain. We assume that the resource allocation is performed for each session $s \in \mathcal{S}_{k}$ and its inter-domain path vector is $\mathcal{P}_{k, s}^{1}=\left[m_{1, k, s}, \ldots, m_{h, k, s} \ldots, m_{M_{k, s}, k, s}\right]$, where $M_{k, s}$ is the number of domains on inter-domain path. Index $h$ is the hop index of domain $m_{h, k, s} \in \mathcal{M}_{k, s} \subset \mathcal{M}$ on the path from source to destination. For example, in Fig. 1, session $s=1$ of SP $k=2$ is traced through domains $\mathcal{P}_{2,1}^{1}=[5,2,1]$ and $m_{1,2,1}=5$ is the first hop $(h=1)$ on the inter-domain path. The inter-domain path connects source and destination nodes when they are located in different domains (Fig. 1). The NSP obtains the inter-domain path for each session based on the minimum cost routing algorithm over matrix $W$. In this way, the slice resources for each session are requested from the domains with the highest availability.

\section{F. Intra-Domain Slice Tracing}

In each domain on an inter-domain path, slice tracing is further refined. An intra-domain path is a result of a multipath routing algorithm that finds all edge-disjoint paths in the domain graph (Fig. 3). The steps of the algorithm for finding all disjoint paths in an undirected graph can be found in [30]. In the access network of the source node of SP $k$ for session $s$ (i.e. in domain $m_{1, k, s}$ ), an intra-domain path is traced between source $U_{k, s}$ and a node that is a gateway to the next domain on the inter-domain path (i.e. $m_{2, k, s}$ ). Similarly, in the access domain of the destination $U_{k, s}^{\prime}$ (i.e. domain $m_{M_{k, s}, k, s}$ ), a path is traced between the gateway from the domain $m_{M_{k, s}-1, k, s}$ and the destination node. In any other domain on the interdomain path, $m_{h, k, s}$, a path is traced between a gateway from domain $m_{h-1, k, s}$ and a gateway to $m_{h+1, k, s}$. We denote individual paths in a multi-path with index $f=1, \ldots, F_{k, s}^{m}$, where $F_{k, s}^{m}$ is the total number of disjointed paths for session $(k, s)$ in domain $m$. An intra-domain path is represented by the set $\mathcal{P}_{h, k, s}^{2}=\bigcup \mathcal{P}_{h, k, s, f}^{2}$, where $\mathcal{P}_{h, k, s, f}^{2}$ represents the set of nodes $i^{m}$ on a path $f$ with $i^{m} \in \mathcal{I}^{P, m}$ and $\mathcal{P}_{h, k, s, f}^{2} \subset \mathcal{I}^{P, m}$ 
TABLE I

TABLE OF NOTATIONS

\begin{tabular}{|c|c|c|c|}
\hline Symbol & Meaning & Symbol & Meaning \\
\hline $\mathcal{M}, M, m$ & Set, number and index of NOs & $\mathcal{K}, K, k$ & Set, number and index of SPs \\
\hline $\mathcal{I}^{m}, I^{m}$ & Set and number of physical nodes of NO $m$ & $\mathcal{L}^{m}, L^{m}$ & Set and number of physical links of NO $m$ \\
\hline $\mathcal{S}_{k}$ & Set of sessions of SP $k$ & $s$ & Index of sessions of SP $k$ \\
\hline$U_{k, s}$ & Source of SP $k$ 's session $s$ & $U_{k, s}^{\prime}$ & Destination of SP $k$ 's session $s$ \\
\hline$d_{k, s}(t)$ & E2E delay of session $(k, s)$ & $d_{k, s}^{m}(t)$ & Delay of session $(k, s)$ in domain $m$ \\
\hline $\mathrm{j}, \mathrm{J}$ & Index and number of inter-domain paths & $1-\epsilon_{k, s}$ & End-to-end reliability of SP $k$ session $s$ \\
\hline $1-\epsilon_{k, s}^{m}$ & Reliability of SP $k$ session $s$ over NO $m$ & $\delta_{k, s}^{m-}$ & Speedup time (credit) by NO $m$ for SP $k$ session $s$ \\
\hline$\delta_{k, s}^{m+}$ & Extra delay (debt) by NO $m$ in NS $k$ session $s$ & $\mathcal{P}_{k, s}^{1}$ & Vector of inter-domain path for SP $k$ session $s$ \\
\hline$C^{m}$ & Average capacity of a domain $m$ & $\mathbf{W}, w_{m, m^{\prime}}$ & Inter-domain weighted topology matrix/element \\
\hline$h$ & Index for hop at the inter-domain path & $m_{h, k, s}$ & Domain on a inter-domain path for SP $k$ session $s$ \\
\hline $\mathcal{P}_{k, s}^{2}$ & Intra-domain path for session $(k, s)$ & $\mathcal{P}_{k, s, f}^{2}$ & Path $f$ of intra-domain path for session $(k, s)$ \\
\hline $\bar{N}_{k, s}, \sigma_{N_{k, s}}$ & Mean and variation of frame size for $(k, s)$ & $\mathcal{F}_{N_{k, s}}(X)$ & Cumulative distribution function (CDF) of frame size \\
\hline$r_{k, s, f}^{m}$ & Rate on path $f$ in NO $m$ for session $(k, s)$ & $d_{k, s, f}^{m}$ & Achievable delay for $r_{k, s, f}^{m}$ \\
\hline $\mathcal{S}^{m}$ & Set of sessions in domain $m$ & $A_{k, s}^{m}$ & Incidence matrix of domain $m$ for sessions $n \in \mathcal{S}^{m}$ \\
\hline$\nabla^{m}, \nabla^{m, l}$ & Vectors of allocated rates & $\theta_{k, s}^{m} / \Delta_{k, s}^{m}$ & Available credit in NO $m$ for session $(k, s)$ \\
\hline $\mathcal{T}_{k, s}^{m}$ & Throughput of the slice & $\mathbf{U}_{k, s}^{m}$ & Utility function of the slice \\
\hline
\end{tabular}

with $m_{h, k, s}=m$. The intra-domain slice tracing is performed by the NSA in each domain. The paths discovered by the tracing algorithm are passed on to the resource allocation algorithm (in Section VB) that determines the optimal rate allocation for each path. A domain slice is traced over multiple paths to allow accommodation of more sessions under limited link capacity by reducing the delay per domain.

\section{G. Domain Slice Stitching Function}

Unlike single domain network slicing, multi-domain network slicing requires forwarding the traffic from one domain to the other. However, end-to-end encapsulation cannot be assumed over different technologies and different domains. Each NSA is allowed to independently select and manage its overlay/isolation method [7]. In our model, the domain gateways adopt the role of domain slice gateways, i.e. logical nodes where the interconnection of slice segments takes place. They perform the stitching function to translate/strip/impose encapsulations. Details of this procedure can be found in [12].

\section{END-TO-END LATENCY AND RELIABILITY}

In this section, we will model end-to-end latency and reliability for message-latency-limited traffic.

\section{A. End-to-End Latency Requirement and Reliability}

The end-to-end delay (E2ED) for a session $s \in \mathcal{S}_{k}$ of SP $k$ is the accumulated processing, queuing, transmission and propagation delays from source $U_{k, s}$ to its destination $U_{k, s}^{\prime}$. For session $(k, s)$, the E2E delay observed over operation time is $d_{k, s}(t)=\sum_{m \in \mathcal{M}_{k, s}} d_{k, s}^{m}(t)$. In order to globally guarantee a cutoff E2ED $D_{k, s}$ for each session $s \in \mathcal{S}_{k}$, we require every domain $m$ in $\mathcal{M}_{k, s} \subset \mathcal{M}$ to locally guarantee a delay $D_{k, s}^{m}$. This means that, for a given E2ED $D_{k, s}$, the network slice is maintained to provide a delay that is under the local delay threshold $D_{k, s}^{m}$ in each domain $m$ so that $\sum_{m \in \mathcal{M}_{k, s}} D_{k, s}^{m} \leq$ $D_{k, s}$.

Each SNS and DSNS of session $(k, s)$ should have enough resources to guarantee that the delay is under the threshold with probability higher than the requested reliability:

$$
\begin{aligned}
& \text { a) } \mathbb{P}\left(d_{k, s}(t) \leq D_{k, s}\right) \geq 1-\epsilon_{k, s} \quad s \in \mathcal{S}_{k} \\
& \text { b) } \quad \mathbb{P}\left(d_{k, s}^{m}(t) \leq D_{k, s}^{m}\right) \geq 1-\epsilon_{k, s}^{m} \quad s \in \mathcal{S}_{k}
\end{aligned}
$$

where $\epsilon_{k, s}$ and $\epsilon_{k, s}^{m}$ are arbitrarily small; $1-\epsilon_{k, s}$ is the requested $\mathrm{E} 2 \mathrm{E}$ reliability and $1-\epsilon_{k, s}^{m}$ is the reliability request at each domain.

\section{B. Latency Equalization}

In a multi-domain scenario, if the domain $m$ cannot guarantee a local delay threshold $D_{k, s}^{m}$, i.e. does not fulfill the condition from the equation (1b), excessive delay can be compensated for in the rest of the path. The local delay threshold $D_{k, s}^{m}$ of NO $m$ can be increased by $\delta_{k, s}^{m+} \geq 0$, referred to as debt, if the other operators in $\mathcal{M}_{k, s}$ can compensate the extra latency $\delta_{k, s}^{m+}$ by transmitting faster (providing the necessary credits). We refer to redefining of the delay requirements and related resource reallocation in domains as latency equalization. DEENS uses latency equalization: 1) in the slicing process for local delay threshold negotiation between the domains in order to increase the number of accepted slices, and 2) in order to handle slice resizing during the operation time due to variation (usage pattern that deviates from the 
traffic prediction) of the traffic and network conditions (e.g. non-stationary capacity of the wireless links and node or link failure). In Section V, a cooperative delay threshold negotiation algorithm will be presented, while in Section VI, we will explain how the resizing process is handled by the vertical and horizontal adjustments of NSs.

\section{Message-Latency-Limited Traffic}

Latency requirements for VR, AR, real-time control and high definition video traffic differ from those for traditional data traffic. As an example, the E2E latency, including video and audio processing for VR applications [31], has to be less than $13 \mathrm{~ms}$, which is the time after which the human eye starts noticing lag [32]. This is the period in which all information of a video frame and corresponding audio should be received and displayed; delay of individual packets within $13 \mathrm{~ms}$ interval is not essential for this service. This fact gives more freedom in communication protocol design as the normalized delay limit per packet does not have to be as strict as 13/(Number of packets per frame) $\mathrm{ms}$ but can tolerate higher delays of certain individual packets as long as the whole frame is received in $13 \mathrm{~ms}$. Transmission of different packets of one frame over parallel paths allows further relaxation of latency requirements and allows higher delay thresholds per packet. Another feature of VR is that the volume of data generated per frame is known. We exploit these two characteristics to define the delay model in the next section. Later, we will generalize our delay model to include other use cases where traffic volume might vary over time from the prediction at the moment of slicing.

\section{Latency Over Multihop Multipath DSNS}

The local source and local destination nodes of session $(k, n)$ in domain $m$ are connected by multiple intra-domain paths as in Fig. 3. Each path $f$ spans over different number of nodes in domain $m$ that will be referred to as the number of intra-domain hops $H_{k, s, f}^{m}$. Depending on the number of hops, packets propagating on different paths with same transmission rates experience different latency. The E2ED requirement for a message consisting of $N_{k, s}$ packets will be considered. We assume that the source generates and buffers the frame before transmission starts and that the processing delay of generating data is not included in the delay threshold $D_{k, s}$. The resources of a slice are dedicated to exclusive use of a session. Therefore, the packet delivery time, i.e. time required for one packet to be sent between two neighbouring nodes, equals the sum of transmission delay and propagation delay. For simplicity, we will assume that packets are of the same size and the propagation delay is negligible.

The packet transmission rate (per second) in path $f$ is denoted as $r_{k, s, f}^{m}$. It is expected from the network that the resources are available at the time the allocation request is made, so there is no congestion on the path associated with an NS [7]. Only faults and/or topology changes can cause failure to meet the service assurances. This implies that the portion of link dedicated for network slice services may not contend with similar service for the life of the connection [7]. Therefore, in this setting, no queue is formed in the nodes.
The requested rate will be met with a guarantee through new and advanced scheduling techniques in the data plane enabled by SDN framework [7]. The number of packets transmitted through path $f$ is $N_{k, s, f}^{m}$ and the following holds:

$$
\sum_{f=1}^{F_{k, s}^{m}} N_{k, s, f}^{m}=N_{k, s} \quad \forall m \in \mathcal{M}_{k, s} .
$$

The time required for $N_{k, s, f}$ packets to be transmitted over path $f$ with $H_{k, s, f}^{m}$ hops is:

$$
d_{k, s, f}^{m}=\frac{H_{k, s, f}^{m}}{r_{k, s, f}^{m}}+\left(N_{k, s, f}^{m}-1\right) \frac{1}{r_{k, s, f}^{m}} .
$$

The total delay of transmitting $N_{k, s, f}^{m}$ packets over multiple paths is equal to the delay of the path that is the longest:

$$
d_{k, s}^{m}=\max \left(d_{k, s, 1}^{m}, \ldots, d_{k, s, F_{k, s}^{m}}^{m}\right) .
$$

For the allocated capacities that enable given rates and number of hops on each path, it is possible to determine how to optimally split traffic among the paths, so the total delay is minimized. We can write the following optimization problem:

$$
\min _{\left[N_{k, s, 1} \ldots N_{k, s, F_{k, s}^{m}}\right]} \max \left(d_{k, s, 1}^{m}, \ldots d_{k, s, F_{k, s}^{m}}^{m}\right) .
$$

Thus we have a set of $F_{k, s}^{m}-1$ linear equations $d_{k, s, f}^{m}=d_{k, s, f^{\prime}}^{m}$ for $\forall f, f^{\prime} \in\left\{1, \ldots, F_{k, s}^{m}\right\}$ that together with (2) give a unique solution to $N_{k, s, f}^{m} \forall f$.

The amount of packets on each path $N_{k, s, f}$ depends on the number of hops and rates over all paths.

For the given delay threshold $D_{k, s}^{m}$ and the number of hops on the path, the required rate can be expressed as $r_{k, s, f}^{m}=$ $\left(H_{k, s, f}^{m}+N_{k, s, f}^{m}-1\right) / D_{k, s}^{m}$. The total effective rate through domain $m$ is then equal to

$$
\begin{aligned}
\sum_{f} r_{k, s, f}^{m} & =\frac{\sum_{f}\left(H_{k, s, f}^{m}+N_{k, s, f}^{m}-1\right)}{D_{k, s}^{m}} \\
& =\frac{N_{k, s}-F_{k, s}^{m}+\sum_{f} H_{k, s, f}^{m}}{D_{k, s}^{m}} .
\end{aligned}
$$

By denoting the total number of hops on all paths of session $(k, s)$ in domain $m$ as $H_{k, s}^{m}$, we have $\sum_{f} r_{k, s, f}^{m}=\left(N_{k, s}-\right.$ $\left.F_{k, s}^{m}+H_{k, s}^{m}\right) / D_{k, s}^{m}$. This means that if the sum of path rates allocated to session $(k, s)$ in domain $m$ is equal to the righthand side of this relation, there is an optimal traffic split that can be obtained from (5), so local delay threshold requirement $D_{k, s}^{m}$ is met. We denote the optimal sum of path rates that achieves the delay threshold as $r_{k, s}^{m, *}$ :

$$
r_{k, s}^{m, *}=\frac{N_{k, s}-F_{k, s}^{m}+H_{k, s}^{m}}{D_{k, s}^{m}} .
$$

In the allocation process, domain $m$ grants resources to session $(k, s)$. The available sum of path rates can be written as

$$
\sum_{f} r_{k, s, f}^{m}=\left\{\begin{array}{l}
r^{\prime}<r_{k, s}^{m, *} \\
r_{k, s}^{m, s} \\
r^{\prime \prime}>r_{k, s}^{m, *}
\end{array} .\right.
$$

If the sum of path rates equals to $r^{\prime}$, not enough resources are allocated, and the achieved delay is longer than the 
threshold. If it equals to $r^{\prime \prime}$, the achieved delay is smaller than the delay threshold, and while the requirement is fulfilled, the network resources are not efficiently used.

\section{E. Reliability of Network Slice for Statistically Known Traffic}

To obtain the relationship between rate (allocated capacity) and delay for limited-latency traffic, so far we have assumed that the number of packets in a frame is constant and that the slicing is done based on the given traffic prediction. However, during the operation time of an NS, the volume of network traffic may vary due to imperfect prediction and/or due to the nature of service itself. This means that, for the allocated sum rate as defined in (7), the resulting E2ED can vary as well. In a general case when the session $(k, s)$ is active, the number of packets in the frame is time-dependent $N_{k, s}(t)$. This is a discrete random variable, and its probability distribution and variance depend on the particular use case. The mean value of frame size is denoted as $\bar{N}_{k, s}$ and the variance as $\sigma_{N_{k, s}}$. From (1) and (7), we can write the reliability condition as follows:

$$
\mathbb{P}\left(\frac{\left(N_{k, s}(t)-F_{k, s}^{m}+H_{k, s}^{m}\right.}{\sum_{f} r_{k, s, f}^{m}} \leq D_{k, s}^{m}\right) \geq 1-\epsilon_{k, s}^{m} .
$$

where $F_{k, s}^{m}$ is the number of paths and $H_{k, s}^{m}$ is number of hops for session $(k, s)$ in NO $m$. After rearranging, we have:

$$
\mathbb{P}\left(N_{k, s}(t) \leq D_{k, s}^{m} \Sigma_{f} r_{k, s, f}^{m}+F_{k, s}^{m}-H_{k, s}^{m}\right) \geq 1-\epsilon_{k, s}^{m}
$$

For the known probability distribution of $N_{k, s}(t)$, the probability above is the cumulative distribution function (CDF) of the number of transmitted packets in a frame $\mathcal{F}_{N_{k, s}}(X)$ :

$$
\mathcal{F}_{N_{k, s}}\left(D_{k, s}^{m} \Sigma_{f} r_{k, s, f}^{m}+F_{k, s}^{m}-H_{k, s}^{m}\right) \geq 1-\epsilon_{k, s}^{m} .
$$

\section{Dynamic End-To-End Network Slicing (DEENS)}

In this section, we present the framework for dynamic end-to-end slicing in a multi-domain network to provide for message-latency-limited service and also define slice throughput and slice efficiency. We start with the assumption that the SPs monitor their traffic to forecast their users' requirements. Then, they use these predictions to request end-to-end NSs from NSP. Since there might be some errors in predictions, the established NSs need to be adjusted during the operation time. This framework dynamically adapts to the current state of the network.

\section{A. Credit and Debt Self-Readjusting Resource Allocation}

We assume that allocation is simultaneously performed for a set of SPs denoted with index $k \in \mathcal{K}$ where the request of each SP consists of a set of sessions with index $s \in \mathcal{S}_{k}$. We assume that the maximum allowed E2ED for session $(k, s)$ is total delay budget $B_{k, s}=(1-\zeta) D_{k, s}$ that the resource allocation algorithm at NSP has available at the beginning of the allocation process. The parameter $\zeta$ is a small number and $1-\zeta$ represents a margin in rate allocation that allows certain variation in traffic under E2ED constraint. After the interdomain path $\mathcal{P}_{k, s}^{1}$ is determined, the NSP sends a resource allocation request to NSA of every domain on this path. The request is formulated in terms of delay since the necessary rate cannot be known to an NSP as it does not know the topology of each domain. Further, we will denote local delay threshold $D_{k, s}^{m}$ as $D_{h, k, s}$ when we want to emphasize the place of domain $m$ in hops on the inter-domain path. The goal of NSP is to split the delay budget $B_{k, s}$ among the domains into local delay budgets $B_{h, k, s}$ in such a way that the local achievable delay does not exceed the given local budget and that $B_{k, s}=\sum_{h=1}^{M_{k, s}} B_{h, k, s}$. Another goal of the resource allocation algorithm is to find an allocation that will maximize the number of connections accepted by the network with guaranteed latency. Therefore, the local delay budgets will not only depend on the resource availability in the network but also on the other requests that network receives. NSA $m$ receives request for all sessions $(k, s)$ that use domain $m$ from their NSPs. Each request contains information on the local source and destination nodes of a session, its local delay budget $B_{k, s}^{m}$ and expected frame size $N_{k, s}$. The resource allocation algorithm is executed in cooperation between NSP and NSA, and in order to perform negotiation of delay budget splitting between the domains, it is executed in 3 phases.

(F1) In the first phase, the budget of each session is equally divided among all $M_{k, s}$ domains on the inter-domain path: $B_{h, k, s}=B_{k, s} / M_{k, s}$, for $\forall h \in\left\{1, \ldots, M_{k, s}\right\}$. Resources are pre-allocated (reserved) based on the request received at NSA. Each domain runs the resource allocation optimization algorithm to share the resources among the requests in a fair manner. At the end of this phase, the rate allocation and the achievable delay are known for each session. The achievable delay can be within the budget if enough resources are allocated to the session, or it can exceed the budget otherwise. Optimization does not allow excess resources to be allocated to any session in order to keep the second phase of the algorithm, budget balancing, simple and fair. F1 represents minimum cost routing and is used as a baseline for performance comparison with our complete three phase algorithm.

(F2) If the E2E achievable delay of a session exceeds the E2ED threshold, with the knowledge of the previously granted resources in each domain, the local budget requests are updated by NSP and the pre-allocation is repeated in the second phase until the adequate local thresholds are found. This is the budget balancing phase, and the details will be presented later in this section.

(F3) If the threshold for a source cannot be achieved in F2, an alternative inter-domain path is found by NSP in the third phase, and pre-allocation is continued.

A session that can acquire sufficient resources during the allocation process, so its achievable delay is within its budget, is accepted by the network. This means that the previously preallocated resources are granted for exclusive use of session's SP. The collection of all resources granted to the SP $k$ becomes network slice $k$. The role of the resource allocation algorithm is to perform slice admission control, i.e. select which sessions can be granted resources by the network and served with required performance. After this process, sessions whose requests are rejected due to lack of resources are dropped. 
The delay $D_{h, k, s}$ at the DSNS in domain $m$ depends on the traffic characteristics and allocated resources to the slice (rate, number of hops and paths). This means that minimum achievable delay will depend on the size of the domain, network technology (maximum transmission rate), and traffic in the domain at the moment of pre-reservation, i.e. available capacity of the domain, and it can vary significantly within domains. If the pre-allocated local budget $B_{h, k, s}<D_{h, k, s}$, it means that not enough resources are available for the connection. For such a connection, we say that it has debt, and the delay budget for that network has to be increased to $B_{h, k, s}^{\prime}=D_{h, k, s}$, while the budget in some other domain has to be decreased by an amount of $B_{h, k, s}-B_{h, k, s}$, which we call credit. In phase 2, it is determined in which domain these additional resources can be acquired in the process of latency equalization. In the following subsections, we present the details of each phase of resource allocation algorithm

\section{B. Optimization of Initial Allocation in a Domain (F1)}

The task of the resource allocation protocol at an NSA is to find an optimal rate allocation for a predetermined intradomain path for each session. Each domain $m \in \mathcal{M}_{k, s}$ receives initial allocation requests from the NSP of each sessions $(k, s)$ traced through domain $m$. We denote the set of these sessions as $\mathcal{S}^{m}$. We form the incidence matrix $\mathbf{A}_{\mathcal{S}^{m}}^{m}$ whose entries depend on whether or not physical link $l \in \mathcal{L}^{m}$ belongs to path $f$. The size of the matrix is $L_{m} X \sum_{\mathcal{S}^{m}} F_{k, s}^{m}$, and each column represents one path of the session $(k, s) \in \mathcal{S}^{m}$. The entries $a_{l^{m}, f_{k, s}}$ of this matrix are defined as

$$
a_{l^{m}, f_{k, s}}= \begin{cases}1 & \text { if path } \mathcal{P}_{h, k, s, f}^{2} \text { uses link } l^{m} \\ 0 & \text { otherwise }\end{cases}
$$

for $h$ such that $m_{h, k, s}=m$.

The rate allocated to session $(k, s)$ on path $f$ is $r_{k, s, f}^{m}$, while the rates allocated to all the paths of session $(k, s)$ are represented by the vector $\mathbf{r}_{k, s}^{m}=\left[r_{k, s, 1}^{m}, \ldots, r_{k, s, F_{k, s}^{m}}^{m}\right]$. Given the set of sessions $\mathcal{S}^{m}$ that are requesting resources from domain $m$, we can write the vector of all path rates allocated in this domain as $\mathbf{r}^{m}=\left[\mathbf{r}_{k, s}^{m} \mid(k, s) \in \mathcal{S}^{m}\right]$. The order of $(k, s)$ sessions in the vector should be the same as the order of sessions in the columns of the matrix $\mathbf{A}_{\mathcal{S}^{m}}^{m}$. In the resource allocation process, domain $m$ assigns rate to each flow of each user. On each link $l^{m} \in \mathcal{L}^{m}$ we let $r^{m, l}$ be the total assigned rate, while vector $\mathbf{r}^{m, l}=\left[r^{m, 1}, \ldots, r^{m, L^{m}}\right]$ represents link rate assignment in the entire domain. The total link rate is obtained as a sum of the rates for the paths that use that link. In the matrix form, we have $\mathbf{A}_{\mathcal{S}^{m}}^{m} \mathbf{r}^{m}=\mathbf{r}^{m, l}$. We assume that the links can be used in both directions and that the total capacity of the link is split between the directions based on the needs. Therefore, the total amount of traffic on each link cannot exceed the available capacity on that link $\mathbf{r}^{m, l} \preceq \mathcal{C}^{m}$. The achievable delay of session $(k, s)$ through domain $m$ for the given rate allocation $\mathbf{r}_{k, s}^{m}$ from (7) equals to:

$$
d_{k, s}^{m}=\left(N_{k, s}-F_{k, s}^{m}+H_{k, s}^{m}\right) /\left(\Sigma_{f} r_{k, s, f}^{m}\right) .
$$

In order to minimize the usage of the resources, we impose an upper limit on the rates as follows:

$$
\Sigma_{f} r_{k, s, f}^{m} \leq\left(N_{k, s}-F_{k, s}^{m}+H_{k, s}^{m}\right) / B_{k, s}^{m} .
$$

Since local budget at domain $m B_{k, s}^{m}<D_{k, s}^{m}$, this represents an upper bound on the allocated rates. It allows the algorithm to share the available capacities fairly among the users and allocate as much rate as possible to each user but not more than necessary to achieve the delay required by the local budget including the margins. As a result of the rate allocation, the achievable delay can either be equal to the local delay budget, or it can be longer if the domain does not have sufficient resources. Our algorithm does not allow achievable delay to be less than budget (upper limit on resources) as this would make second phase of the algorithm inefficient.

Our goals in the resource allocation process are to minimize the delay for all sessions and to split available domain capacities in such way that as many sessions as possible are admitted. Therefore, the objective function of the optimization problem is the sum of achievable delays of all sessions $(k, s) \in \mathcal{S}^{m}$. The resource allocation for message-latency-limited users in a domain $m$ is formulated as the following optimization problem:

$$
\underset{\mathbf{r}^{m} \mathbf{r}^{m, l}}{\operatorname{minimize}} \sum_{(k, s) \in \mathcal{S}^{m}} d_{k, s}^{m}
$$

subject to

$$
\begin{aligned}
& \text { 1) } \mathbf{A}_{\mathcal{S}^{m}}^{m} \mathbf{r}^{m}=\mathbf{r}^{m, l}, \quad \mathbf{r}^{m, l} \preceq \mathcal{C}^{m, l} \\
& \text { 2) } \sum_{f} r_{k, s, f}^{m} \leq\left(N_{k, s}-F_{k, s}^{m}+H_{k, s}^{m}\right) / B_{k, s}^{m}, \quad \forall(k, s) \in \mathcal{S}^{m} \\
& \text { 3) } \mathbf{r}^{m} \succeq 0, \quad \mathbf{r}^{m, l} \succeq 0 .
\end{aligned}
$$

The variables in this optimization problem are $\mathbf{r}^{m}$ and $\mathbf{r}^{m, l}$. The first and second constraints are for the requirement that the allocated rates should be less than or equal to the available capacity of a domain. The third constraint means that a domain should not allocate more capacity to the network than required to achieve the given local budget, including the margins. This is an upper limit for the allocated capacity, and it prevents the network from allocating unnecessary large capacity to one request. However, the allocation process allows the delay to be slightly shorter than the budget for the amount of delay budget margin $1-\zeta$. The last two constraints are due to the fact that all allocated rates should be non-negative real values.

The objective function is a function of optimization variable r. However, the problem in this form is not convex. In order to make it convex, we have to introduce $d_{k, s}^{m}$ as an auxiliary variable and an additional condition that:
a) $d_{k, s}^{m} r_{k, s}^{m}=N_{k, s}-F_{k, s}^{m}+\Sigma_{f} H_{k, s, f}^{m}$
b) $r_{k, s}^{m}=\Sigma_{f} r_{k, s, f}^{m}$.

Hyperbolic constraint (16-a) can be rewritten as a second-order cone program (SOCP) constraint. We use the fact that any relation of the form $z^{2} \leq x y$ can be transformed into

$$
\left\|\left[\begin{array}{c}
2 z \\
x-y
\end{array}\right]\right\|_{2} \leq x+y
$$


where $\|*\|_{2}$ is $l_{2}$ norm. The optimization problem in (15) is then equivalent to:

$\underset{\mathbf{r}^{m} \mathbf{r}^{m, l}, d_{k, s}^{m}}{\operatorname{minimize}} \sum_{(k, s) \in \mathcal{S}^{m}} d_{k, s}^{m}$

subject to

$$
\begin{aligned}
& (15.1), \quad(15.2), \quad(15.3) \quad \text { and } \\
& \left\|\left[\begin{array}{c}
2 \sqrt{N_{k, s}-F_{k, s}^{m}+H_{k, s}^{m}} \\
d_{k, s}^{m}-r_{k, s}^{m}
\end{array}\right]\right\|_{2} \leq d_{k, s}^{m}+r_{k, s}^{m} \forall(k, s) \in \mathcal{S}^{m} \\
& d_{k, s}^{m}>0, \quad r_{k, s}^{m}=\sum_{f} r_{k, s, f}^{m}, \quad \forall(k, s) \in \mathcal{S}^{m} .
\end{aligned}
$$

The problem in (18) is convex and can be solved using standard convex optimization techniques. For the optimal solution, the equality will hold in the SOCP constraint.

After pre-allocation in each domain, the remaining available link capacities are updated so that the reserved resources are retracted from the updated values $C^{m, l}=C^{m, l}-r^{m, l} \quad \forall l^{m} \in$ $L^{m}$ for $\forall m \in M$.

\section{Credit and Debt Trading Mechanism - Budget Balancing (F2)}

After phase F1 is completed, the achievable delays represent current delay thresholds for each domain on the path and we can write $\mathbf{D}_{k, s}=\left[D_{1, k, s}=d_{1, k, s}, \ldots, D_{h, k, s}=\right.$ $\left.d_{h, k, s}, \ldots, D_{M_{k, s}, k, s}=d_{M_{k, s}, k, s}\right]$. The NSP of each session checks if sufficient resources are allocated in all domains, i.e. if $D_{h, k, s}=B_{h, k, s}$. If this is not the case and $D_{h, k, s}>B_{h, k, s}$, the session has debt and the total delay is over budget. The NSP of each session with debt needs to find the domain where it is possible to achieve a smaller delay, so the total delay stays under the desired E2ED threshold. The total debt of one source is $\delta_{k, s}^{(0)}=\sum_{h \in \mathcal{P}_{k, s}}\left(D_{h, k, s}^{(0)}-B_{h, k, s}^{(0)}\right)=D_{k, s}-B_{k, s}^{(0)}$, where (0) denotes values at the beginning of phase F2. Starting from $h=1$ on the path, the source looks for the domain without debt and updates its budget as follows:

$$
B_{h, k, s}^{(1)}=B_{h, k, s}^{(0)}-\delta_{k, s}^{(0)} .
$$

The remaining resources in domain $m_{h=1, k, s}$ that are not prereserved to other sources should then be allocated by domains NSA to source $(k, s)$ using (18) with updated local budget from (19). From here $D_{k, s}^{(1)}$ is obtained and again two cases are possible: either the requirement is met, and the allocation algorithm ends, or the achieved delay is over budget. In the latter case, the debt $\delta_{k, s}^{(1)}$ is calculated and the next domain on the path without the debt is asked for additional resources. Note that, $D_{k, s}^{(1)} \leq D_{k, s}^{(0)}$ and the iterations, denoted by index $i$, are repeated until debt $\delta_{k, s}^{(i)}=0$ or the end of the path is reached. When sufficient resources are acquired, the prereserved resources are allocated to SP $k$. If the allocation is unsuccessful, the NSP executes the inter-domain slice tracing algorithm in search for an alternative path, i.e. phase $\mathbf{F 3}$ is triggered. Steps in F1 and F2 are summarized in Algorithm 1.

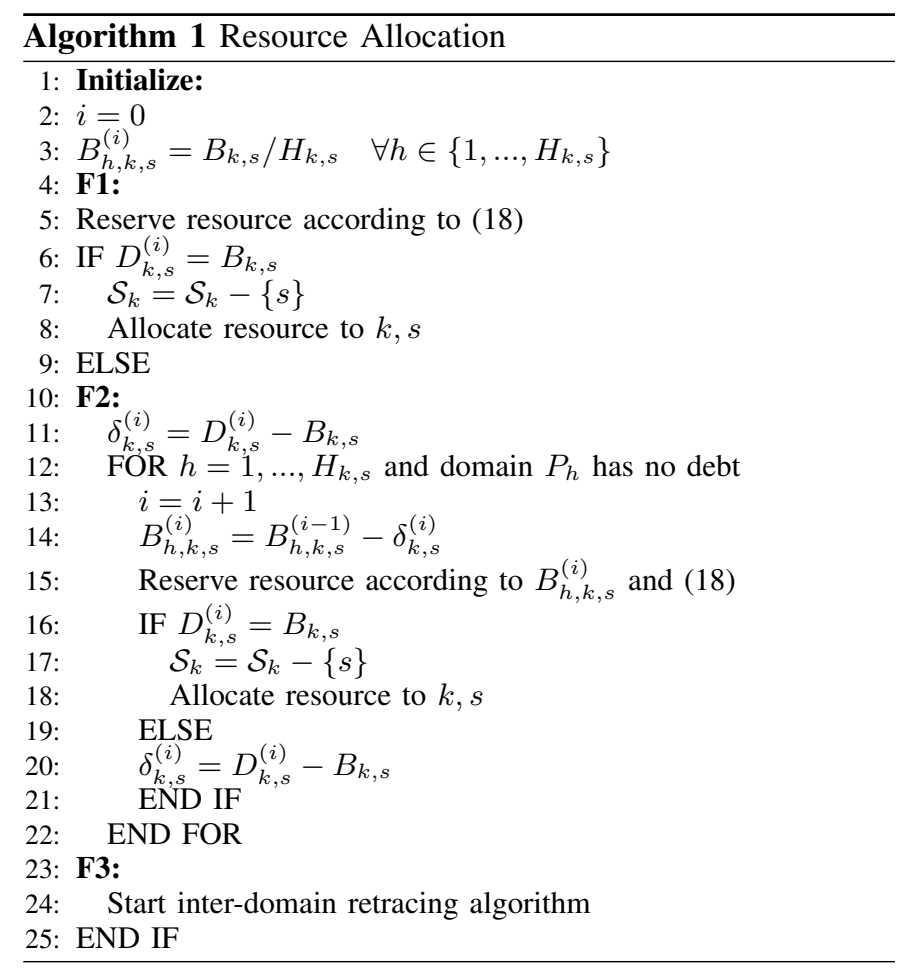

\section{Inter-Domain retracing $(\boldsymbol{F} 3)$}

After phase $\mathbf{F} 2$ of the allocation algorithm is performed, in order to efficiently obtain resources for the remaining sessions in the allocation process, alternative additional inter-domain paths are found by NSPs. These are the sessions that have obtained a certain amount of resources on the first interdomain path $(j=1)$, but their E2E achievable delay is greater than the E2ED budget $d_{k, s}^{(j=1)}=\sum_{m \in M_{k, s}^{(j, 1)}} d_{k, s}^{m,(j=1)}>B_{k, s}$, where index $j$ denotes inter-domain path. If the total number of packets in the frame is $N_{k, s}$, the number of packets from the same frame that can be sent through inter-domain path $j$ is denoted as $N_{k, s}^{(j)}$, and for available rates $r_{k, s}^{(m, *)}$ it can be obtained from the fact that the E2E achievable delay should be equal to the E2ED budget:

$$
\sum_{m \in M_{k, s}^{(j)}} d_{k, s}^{m,(j)}=\sum_{m \in M_{k, s}^{(j)}} \frac{N_{k, s}^{(j)}-F_{k, s}^{m}+H_{k, s}^{m}}{r_{k, s}^{m, *}}=B_{k, s}
$$

After rearranging, we have:

$$
N_{k, s}^{(j)}=\frac{B_{k, s} \sum_{m \in M_{k, s}^{(j)}} r_{k, s}^{m, *}-\sum_{m \in M_{k, s}^{(j)}} H_{k, s}^{m}-F_{k, s}^{m}}{M_{k, s}^{(j)}} .
$$

Once $J$ additional paths are created, in the case that there is still remaining overflow traffic that could not been transmitted within delay bounds, the allocation request can be sent to the path $(\mathrm{J}+1)$ with the updated frame size:

$$
\mathbf{N}_{k, s}^{(J+1)}=N_{k, s}-\sum_{j=1}^{J} N_{k, s}^{(j)}
$$

and the same delay budget value $B_{k, s}$. An alternative path should avoid a domain where the session $(k, s)$ is having debt, as it is not possible to obtain more resources for this session. The weights of inter-domain links towards a congested domain 
should be set to infinity. The link weights to other domains should be updated to reflect the general resource availability $\bar{C}^{m}$ in the domain after $\mathbf{F} 2$ allocation, based on remaining link capacities. After obtaining the new minimum cost route $\mathcal{P}_{k, s}^{1,(j)}$, the allocation process for the remaining traffic of the session is performed following the same steps of F1 and F2 as before. This process is summarized in Algorithm 2.

The session accumulates resources during the allocation process for each new inter-domain path until enough resources are acquired. The allocation process for session $(k, s)$ can be unsuccessful in following cases:

1) if the session runs out of the options for alternative paths before acquiring sufficient resources;

2) if no resources are acquired in two consequent interdomain paths;

3 ) if the total number of parallel intra-domain flows becomes too large for the frame size $N_{k, s}$ to be split.

In any of these above situations, the allocation algorithm for session $(k, s)$ ends.

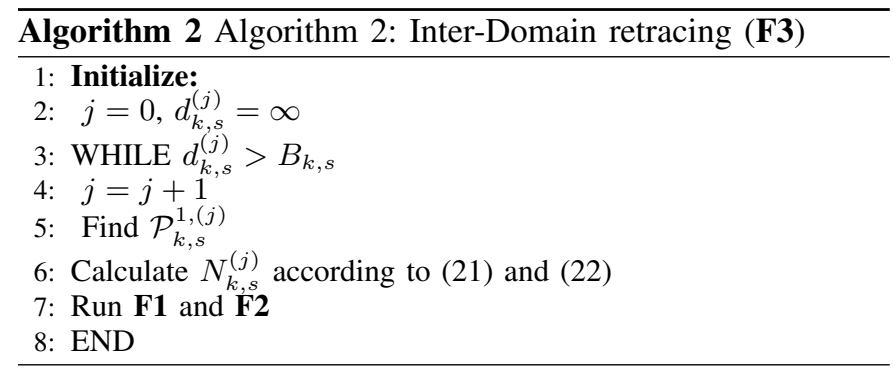

\section{E. Slice Throughput and Utility Function}

A slice is allocated to session $(k, s)$ based on the known frame size $N_{k, s}$ and chosen value of delay budget margin parameter $\zeta_{k, s}^{m}$. We define throughput of a slice as a percentage of successfully transmitted traffic, i.e. where the delay requirement is met. Using the left hand side of equation (9), i.e. (11), which is the expression for the probability of successful transmission, we can define slice throughput as:

$$
\mathcal{T}_{k, s}^{m}=N_{k, s} \mathcal{F}_{N_{k, s}}\left(D_{k, s}^{m} \sum_{f} r_{k, s, f}^{m}+F_{k, s}^{m}-H_{k, s}^{m}\right) .
$$

For a larger margin parameter, the throughput of the slice with traffic variations is increasing. However, by choosing a very large margin parameter, the resources of the slice might be underutilized. In order to design a robust but economical system, the margin parameter should be carefully chosen. We define the slice utility function or slice efficiency as follows:

$\mathbf{U}_{k, s}^{m}=\left(1-\zeta_{k, s}^{m}\right) N_{k, s} \mathcal{F}_{N_{k, s}}\left(D_{k, s}^{m} \sum_{f} r_{k, s, f}^{m}+F_{k, s}^{m}-H_{k, s}^{m}\right)$

\section{RESIZING OF NS}

The network slices are created based on the SPs prediction of traffic characteristics. During the operation time, the network conditions might change, or the NS can become inadequate for the instantaneous traffic needs. Resources can either be insufficient or underutilized. In order to cope with the network dynamics, the NSs for the SPs can be dynamically adjusted using the concept of horizontal and vertical adjustment.

The established NS $k$ for session $s$ has the delay threshold list $\mathbf{D}_{k, s}=\left[D_{k, s}^{1}, D_{k, s}^{2}, \ldots, D_{k, s}^{m}, \ldots, D_{k, s}^{M}\right]$ where $D_{k, s}^{m}=0$ for $m \notin \mathcal{M}_{k, s}$ as the session does not use those domains. Assume that the achievable delay of session $(k, s)$ in NO $m$ passes the threshold $\mathrm{D}_{k, s}^{m} b y \delta_{k, s}^{m+}$. In order to compensate for the extra delay $\delta_{k, s}^{m+}$, there exist two different credit trading processes.

1) Every domain $m^{\prime} \in \mathcal{M} \backslash m$ hosting session $(k, s)$ can provide horizontal credit $\delta_{k, s}^{m, m^{\prime}-}$ to domain $m$ by transmitting faster.

2) SP $k^{\prime}$ can cut some resources of session $s^{\prime}$ in domain $m$ to provide vertical credit $\Delta_{k^{\prime}, k, s^{\prime}, n}^{m-}$ for session $(k, s)$ in domain $m$.

To determine whether a session $s$ can trade credit either vertically or horizontally, we redefine $\theta_{k, s}^{m}(t)$ as available credit in domain $m$ by $\theta_{k, s}^{m}(t)=D_{k, s}^{m}-d_{k, s}^{m}(t)-\vartheta_{k, s}^{m}$, where $\vartheta_{k, s}^{m}$ denotes the marginal credit to support users' requirements. This value is updated based on the requested delay and reliability. According to the network state in domain $m, \theta_{k, s}^{m}(t)$ can be defined as either debt $\left(\theta_{k, s}^{m}(t) \leq 0\right)$ or credit $\left(\theta_{k, s}^{m}(t)>0\right)$. This credit/debt can be traded/compensated vertically and horizontally by cooperation between SPs and NOs, respectively.

\section{A. Horizontal Credit/Debt Trading}

If session $(k, s)$ is in debt only in domain $m$, the available credits in other domains should be updated by horizontal credit trading process to obtain $\mathbf{D}_{k, s}^{\prime}=\left[D_{k, s}^{1}-\delta_{k, s}^{m, 1-}, D_{k, s}^{2}-\right.$ $\delta_{k, s}^{m, 2-}, \ldots, D_{k, s}^{m}+\delta_{k, s}^{m+}, D_{k, s}^{m+1}-\delta_{k, s}^{m,(m+1)-}, \ldots D_{k, s}^{M_{k}}-$ $\left.\delta_{k, s}^{m, M_{k}-}\right]$ such that the E2ED $D_{k, s}$ is guaranteed. In the vector above, $\delta_{k, s}^{m, m^{\prime}-} \geq 0, \forall m, m^{\prime} \in \mathcal{M}_{k, s}$ and

$$
\sum_{m^{\prime} \in \mathcal{M}_{k} \backslash m}\left(D_{k, s}^{m^{\prime}}-\delta_{k, s}^{m, m^{\prime}-}\right)+D_{k, s}^{m}+\delta_{k, s}^{m+} \leq D_{k, s} .
$$

Let $\mathcal{M}_{k, s}^{-} \subset \mathcal{M}_{k, s}$ and $\mathcal{M}_{k, s}^{+} \subset \mathcal{M}_{k, s}$ denote the sets of domains transmitting faster than thresholds and generating longer delays for session $s$, respectively. Generally, after a successful NS-resizing process (horizontal credit trading process), the sum of the obtained credits should be larger than the sum of debts:

$$
\sum_{m \in \mathcal{M}_{k, s}} \theta_{k, s}^{m}(t) \geq \sum_{m \in \mathcal{M}_{k, s}} \delta_{k, s}^{m+} .
$$

The session $s$ of SP $k$ is horizontally stable if and only if the constraint in (26) holds after the HCT process.

\section{B. Vertical Credit/Debt Trading}

An NO $m$ can locally compensate for debt of a session by trading credit between different active sessions hosted by $m$. Let $\mathcal{S}_{k}^{m}$ denote all active sessions of SP $k$ in NO $m$. Session $(k, s)$ of SP $k$ can participate in vertical credit trading process, if and only if it has remaining credits on the horizontal axis:

$$
\hat{\theta}_{k, s}(t)=\sum_{m^{\prime} \in \mathcal{M}_{k, s}} \theta_{k, s}^{m^{\prime}}(t)-\sum_{m^{\prime} \in \mathcal{M}_{k, s}} \delta_{k, s}^{m^{\prime}+}>0
$$


where $\hat{\theta}_{k, s}(t)$ denotes the maximum credit, which can be shared by session $s$.

The vertical credit trading among sessions $s^{\prime}$ of SP $k$ and all sessions of SPs $k^{\prime}$ in domain $m$ will compensate debt of session $s$ of SP $k$ if the following conditions hold:

$$
\begin{aligned}
& \text { a) } \sum_{k^{\prime} \in \mathcal{K}_{m}} \sum_{s^{\prime} \in \mathcal{S}_{k^{\prime}}^{m}, s^{\prime} \neq n} \Delta_{k^{\prime}, k, s^{\prime}, s}^{m-} \geq D_{k, s}^{m}+\delta_{k, s}^{m+} \\
& \text { b) } \hat{\theta}_{k^{\prime}, s^{\prime}}(t) \geq \Delta_{k^{\prime}, k, s^{\prime}, s}^{m-} .
\end{aligned}
$$

The second constraint guarantees that the transferred credit is less than the available credit of session $s^{\prime}$ in domain $m$.

\section{Complexity of DEENS and Signaling Overhead}

The number of exchanged signaling messages between one NSP and NSAs to update the topology matrix is proportional to the number of domains $M$. To set up the slice for session $(k, s)$, the volume of overhead traffic exchanged between the NSP and the NSAs is proportional to the number of interdomain hops $M_{k, s}$. Measurements on the Internet concluded that in the United States about $96 \%$ of paths have less than 3 hops counted in autonomous systems (i.e. domains) [33]. The number of messages that an NSA exchanges with the domain nodes' for link capacity updates and slice set up are proportional, respectively, to the number of nodes $I^{m}$ and the number of hops on the intra-domain path per session $H_{k, s}^{m}$. The number of hops per session on Internet is measured in [33], [34] and it is found that one session on average has between 5 and 20 hops. Alternatively, we can use the small world approximation [35] for the average number of hops for one session $(k, s)$ is $\sum_{m \in \mathcal{M}_{k, s}} \log \left(I^{m}\right)$. The volume of overhead traffic in the network will further depend on the implementation of the control network that can be inbound, using data network infrastructure, or outbound, having dedicated infrastructure.

Splitting the network into autonomously controlled domains significantly reduces the complexity of path finding. The interdomain minimum cost route can be found by using the Dijkstra algorithm with complexity $O\left(M^{2}\right)$ [36] where each domain represents the vertices of the graph. The complexity of finding all disjointed paths inside a domain is of $O\left(I^{m} L^{m}\right)$ [30]. Assuming that the number of inter-domain paths for a session is $J$, the total complexity of finding a path for a session is of $O\left(J *\left(M^{2}+\max _{m \in \text { ax }}\left(I^{m} L^{m}\right)\right)\right.$. In the case the network is not partitioned into domains, the complexity of finding disjointed paths in the whole network would be of $O\left(\sum_{m} I^{m} * \sum_{m} L^{m}\right)$. For example, if the network has $M=5$ domains with $|E|=8$ inter-domain links, and each domain has $I^{m}=20$ nodes and $L^{m}=30$ links $\forall m$, a centralized solution would have complexity proportional to $5 * 20+5 * 30=15000$. We assume that in the worst-case scenario of the proposed slice admission control, there can be $J=3$ inter-domain paths. The complexity of our proposed solution in this example for one inter-domain path is proportional to $5^{2}+20 * 30=625$, i.e. 1875 for three paths. This means, the complexity of path finding is reduced between 8 and 24 times.

\section{NuMERICAL RESULTS}

In this section, the performance of the proposed dynamic slicing mechanism is evaluated by simulations and numerical analysis using MATLAB. First, we provide results on the performance of the resource allocation algorithm for message limited latency traffic. Then, we study NS reliability.

\section{A. Resource Allocation}

We assume that the network is divided into $M=5$ domains. The topology of the network is given in Fig. 1. Each domain has random topology with $I^{m}=10$ nodes. Link capacities $\mathcal{C}^{m}$ are randomly generated from the uniform distribution between $[50,150]$ packets/second. We choose this range for the purpose of illustrating the trading mechanism in the network with the scarce resources. The network is sliced for $K=2$ SPs each requesting 2 sessions with the same delay requirements. Required delay threshold and the E2E delay budget of each session is $B_{k, s}=10 \mathrm{~ms}$. Positions of the source and destination users, i.e. domain nodes that provide access to the network for source and destination users, are randomly generated within the domains indicated in Fig. 1. To simplify the interpretation of the results, we first assume that $\zeta_{k, s}^{m}=0$ for every $m$. The simulation results show that there is no gap between solutions to optimization problems (15) and (18). The constraint (18) relating delay and rate holds with equality, and, therefore, the obtained optimum solution is at the same time the optimum solution of (15). The achievable delay after initial reservation of resources and E2ED budget for each session is presented in Fig. 4. We observe that only session $k=2, s=2$ can achieve the required delay after initial allocation in F1, i.e. delay equals to its E2ED budget. For other sessions, since the achievable delay exceeds the delay budget, the resource allocation algorithm for these sessions has to enter stage F2 in order for them to acquire sufficient resources. For session $k=1, s=1$, we can see in Fig. 5 how the delay budget and the achievable delay break down into local budget and local achievable delay in each domain along the path. The allocation has debt in $m=1$ and $m=2$, which are the first and second domains on the path. In the domain $m=5$ on the path, the requirements are fulfilled. In the $\mathbf{F} 2$ phase, request for additional resources will be sent to $m=5$ as it is the only domain without debt.

Fig. 5 shows the results of the $\mathbf{F} 2$ stage for session $k=$ $1, s=1$. It can be observed that the entire debt of the session is successfully covered by the credit obtained in domain $m=5$ to compensate for the debts in domains 1 and 2 . The delay budget of this session is balanced, and the session is allocated necessary resources. In Fig. 4, the results after completion of F2 are presented for all the sessions. Session $k=1, s=2$ also successfully obtains enough resources and its achievable delay is in balance with its delay budget. Session $k=2, s=1$ is compensated for its credit partially. However, this session is still in debt and the allocation process continues in phase F3. In the retracing process, a second inter-domain path is found $\mathcal{P}_{2,1}^{1,(2)}=[5,3,1]$ for this session. The new path goes through the domains $m=5,3$, and 1 . The budget for the second path is equal to the budget in the first path (Fig. 6), while in order 
to eliminate debt in the first inter-domain path only $83 \%$ of the traffic should be sent on it. For the remaining $17 \%$ of the traffic, resources are requested on the second inter-domain path.

Notice that there are several factors contributing to the availability of resources in a domain viewed from the perspective of a session: a) the initial available link capacities on the path the session is utilizing; b) the transmission rate that is sufficient to provide desired delay in the domain with specific number of parallel paths and hops; and c) link capacities fairly shared between the competing sessions if their paths happen to use the same link. All of these result in some domain not having enough resources to provide requested delay for a session, while others have sufficient resources and even excess resources that can be allocated in F2 and F3. In Fig. 6 , it can be seen that after repeated allocations, resources are successfully assigned to this session, and the achievable delay on the second path is equal to the delay budget, i.e. the entire remaining traffic can be sent on the second path. Since no additional paths are necessary, the dynamic end-to-end network slicing (DEENS) algorithm ends at this point. Next, we investigate performance of our algorithm with different number of domains, domain sizes and number of sessions. We compare two different network topologies, with $M=5$ domains in Fig. 1 and $M=8$ domains in Fig. 8. We define the following scenarios: $\mathbf{S} 1 M=5, K=2, S_{k}=2, I^{m}=10$; S2 $M=8, K=2, S_{k}=4, I^{m}=10 ; \mathbf{S} 3 M=5, K=$ $2, S_{k}=2, I^{m}=20 ; \mathbf{S} 4 M=5, K=2, S_{k}=4, I^{m}=10$. Other parameters are same as before, while the positions of users are randomly generated taking into account that source and destination should not be in the same domain. In Fig. 7 we show percentage of sessions successfully allocated in each phase of the algorithm for scenarios $\mathbf{S} 1-4$. As the number of domains increases, $\mathbf{S} 1$ compared to $\mathbf{S} 2$, and consequently size of the network increases, contention among different sessions for the resources decreases. Therefore more resources are allocated in phase F1. Note that number of sessions is also increased in $\mathbf{S} 2$ otherwise no competition between the sessions would occur. With the increase in the domain size, $\mathbf{S} 1$ compared to $\mathbf{S} 3$, competition between session for the resources decreases and more sessions can be allocated sooner. In scenario $\mathbf{S} 4$, the number of sessions is higher than in $\mathbf{S} 1$. Due to increased competition among sessions and also scarcity of network resources not all requests can be accepted. However, the benefits of latency equalization mechanism are higher than in $\mathbf{S} 1$.

\section{B. Comparison with Existing Schemes}

As it has been discussed earlier, solution of the multidomain slicing problem has to be decentralized. For this reason we compare our solution to a decentralised baseline algorithm rather than the globally optimal solution. Minimum cost routing algorithm with constrained latency is a classical solution proposed in [23] [28] [29]. They all assume delay to be a link parameter and do not consider the relation between the allocated rate and the achieved delay. Therefore, their schemes cannot be directly compared to ours. In order to emphasise improvements achieved by our latency equalization mechanism we compare our results to a decentralised minimum cost routing algorithm with predefined latency requirements per domain. This baseline is equivalent to phase F1 of our algorithm. In the previously considered example allocation, phase F1, from Fig. 4, results in only one session being successfully allocated. Our algorithm finds delay constrained paths for all four sessions after completion of phase F3. Hence, latency equalization shows significant improvement compared to the baseline.

\section{Analysis of Reliability}

We investigate the dynamics of network traffic for which the allocated rate does not violate the reliability condition (11) and also the target rate/budget margin in allocation for the known statistics of the traffic. For this, we approximate the discrete random frame size $N_{k, s}$ by a continuous Erlang distribution. The mean value and the variance of the distribution can be expressed in terms of the shape $\kappa$ and rate $\lambda$ parameters as follows: $\bar{N}=\kappa / \lambda$ and $\sigma=\kappa / \lambda^{2}$. The CDF for the Erlang distribution has the form: $\mathcal{F}(X)=1-\sum_{j=0}^{\kappa-1}\left(e^{-\lambda X}(\lambda X)^{j}\right) / j$ ! where in our case $X=D_{k, s}^{m} \sum_{f} r_{k, s, f}^{m}+F_{k, s}^{m}-H_{k, s}^{m}$. We consider session $(k, s)$ that should achieve delay $D_{k, s}^{m}=1$ ms in domain $m$ with two paths $F_{k, s}^{m}=2$ and number of hops $H_{k, s}^{m}=5$. For the first set of results, we assume that $\sigma_{N_{k, s}}=1$. The value of CDF in (11) as a function of mean frame size is shown in Fig. 9 for different domain sum rates. According to (11), the CDF should be greater than $1-\epsilon_{k, s}^{m}$ to achieve the desired reliability. From the figure, for desired reliability (number close to 1), it can be seen what values of mean frame size are supported by each sum rate.

Next, we consider how an increase in the sum rate affects the achievable reliability for different values of mean frame size and different values of frame size variance. The CDF as a function of sum is given in Fig. 10. For the desired $\epsilon_{k, s}^{m}$, the range of sum rates that the satisfies condition for given traffic parameters can be obtained. The sum rate in our allocation algorithm is determined based on delay budget and assumed average frame size, equation (14) taken with equality. The delay budget for a given delay requirement depends on the margin parameter $\zeta_{k, s}^{m}$. In Fig. 11, we investigate the impact of the choice of margin parameter on reliability. We again assume that the mean frame size is $\bar{N}_{k, s}=30$ packets while different values of variance are given by different curves in the plot. With an increase in variance of frame size, higher margin and consequently higher rate are necessary to maintain the same level of reliability.

Throughput as defined in (23) is presented in Fig. 12 as the function of variation of the frame size $\sigma_{k, s}$ with mean frame size and delay budget margin as a parameter. The sum rate is obtained as in (14) by setting the mean frame size as the frame size parameter. With an increase in traffic variations, i.e. variance of frame size, the throughput decreases. However, with an increase in the margin parameter, the throughput increases.

Next, we assume that the allocation of the slice is done based on the same predicted value of frame size $N_{0}=30$ 


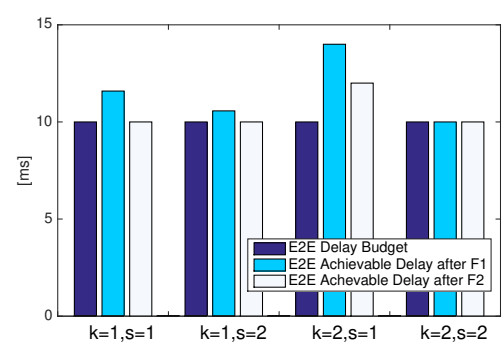

Fig. 4. E2ED budget and achievable delay after completion of phases F1 and F2.

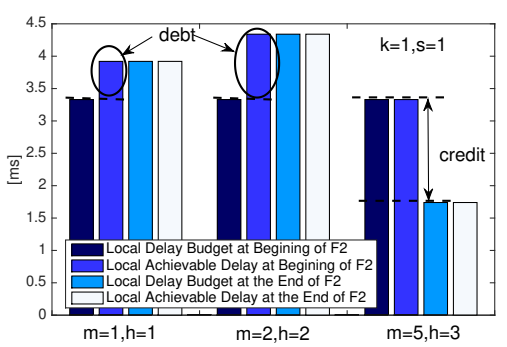

Fig. 5. Local delay budgets and achievable delays at the beginning and end of phase $\mathbf{F 1}$ for session $s=1$ of SP $k=1$ in each hop of inter-domain path.

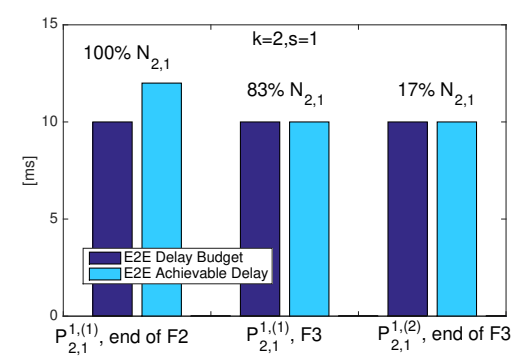

Fig. 6. E2ED budgets and achievable delays before and after completion of phase $\mathbf{F} \mathbf{3}$ for session $s=1$ of SP $k=2$.

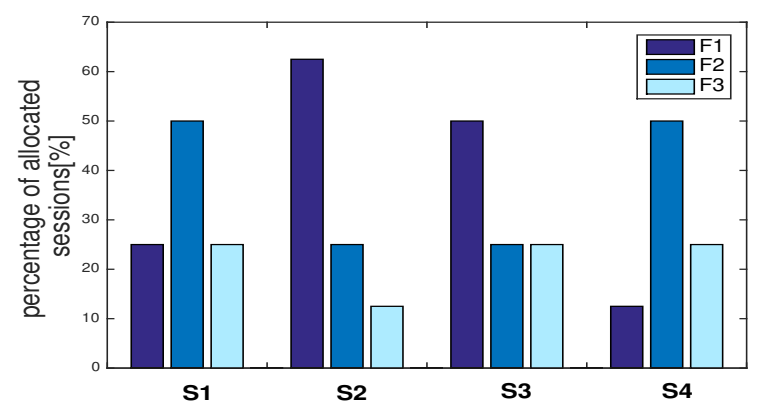

Fig. 7. Percentage of sessions successfully allocated in each phase.

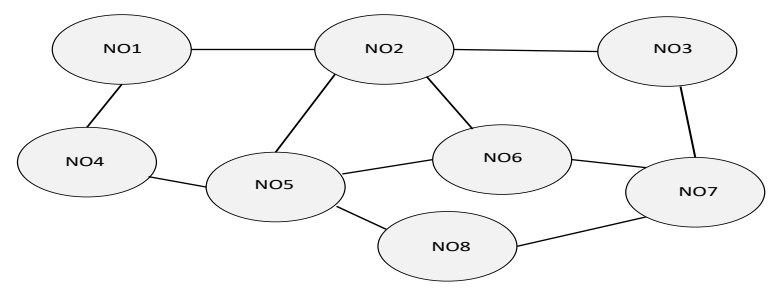

Fig. 8. Network topology with $M=8$ domains

packets and our choice of the margin parameter. During the operation time, the frame size may not only vary around some mean value, but the mean value itself may be changing. We assume large variations of traffic $\sigma_{k, s}=120$, and in Fig. 13 , we investigate how the choice of the margin parameter influences the efficiency of the slice in different ranges of the mean values. The efficiency has a maximum value for each margin value with respect to mean frame size. This means that if the traffic volume increases too much, the system cannot guarantee the delay, and the utilization declines. If the traffic volume decreases, the slice is underutilized.

In Fig. 14, the slice utility is presented as a function of mean frame size and delay budget margin parameter for $\sigma_{k, s}=120$ and $N_{0}=30$. For a sufficiently large margin parameter, any range of the traffic can be supported, even for the large traffic variations as in this example.

\section{CONCLUSION}

This paper proposes a framework for guaranteeing end-toend message-latency-limited communications throughout the multi-domain network. A novel multipath multihop network slicing mechanism has been developed to cope with network dynamics and guarantee end-to-end delay requirements of users. In order to efficiently update the network slices, slicing has been implemented as a two-level slice tracing algorithm in addition to a retracing mechanism. We have proposed an inter-domain routing method based on the average availability of the domain. In addition, we have developed a flexible credit and debt-based resource allocation scheme for network delay equalization over a multihop heterogeneous network. It allows longer than average delays (debt) in certain domains if there is a possibility to compensate (credit) such excessive delays in other segments of the network that can transmit the messages with less latency. This approach increases the number of accepted slices in the initial slice deployment while minimizing usage of the resources and strictly keeping the latency under required threshold. During operation of the slice, vertical and horizontal latency equalizations are used in order to adapt to the traffic dynamics in the network. Through numerical results, we have shown that, with the knowledge of the traffic statistics, network resources can be sliced to achieve the desired end-to-end latency and reliability. As a baseline for performance comparison, minimum cost routing with latency constraints were used. It was shown that this approach enables significantly better utilization of the network resources measured in number of slices with the same latency requirements that can be accommodated in the network. In general, the gain would depend on the network topology, size of the network, traffic volume and temporal occupancy of the physical network.

\section{REFERENCES}

[1] NGMN Alliance, "5G white paper," Next generation mobile networks, white paper, 2015.

[2] Docomo NTT, "DOCOMO 5G white paper," 2014.

[3] Recommendation ITU-T Y.3111 IMT-2020 network management and orchestration framework.

[4] C. Liang and F. R. Yu, "Wireless network virtualization: A survey, some research issues and challenges," IEEE Communications Surveys \& Tutorials, vol. 17, no. 1, pp. 358-380, 2015.

[5] N. M. K. Chowdhury and R. Boutaba, "Network virtualization: State of the art and research challenges," IEEE Communications magazine, vol. 47, no. 7, 2009.

[6] K. Bernardos and et al., "Multi-domain network virtualization,draftbernardos-nfvrg-multidomain-05," https://datatrackerietf.org/doc/draftbernardos-nfvrg-multidomain/, September 2018. 


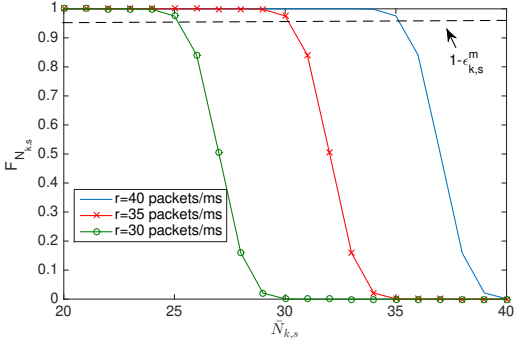

Fig. 9. CDF of number of transmitted packets versus average frame size for $\sigma_{N_{k, s}}=1$.

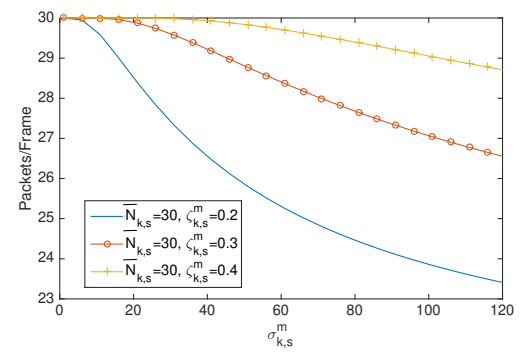

Fig. 12. Throughput versus variation of frame size, when sum rate is obtained for known mean value of frame size and delay budget margin.

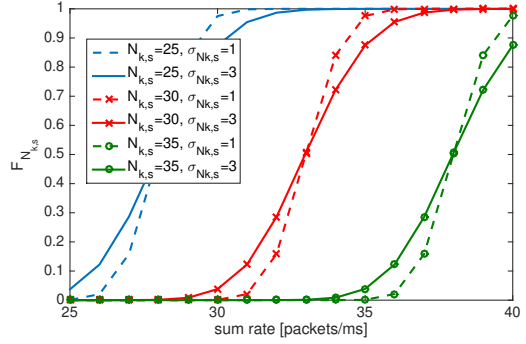

Fig. 10. CDF of number of transmitted packets versus sum rate.

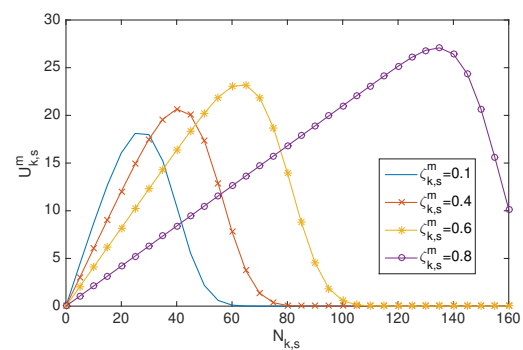

Fig. 13. Efficiency of slice resources versus mean frame size for variation $\sigma_{k, s}=120$ and $N_{0}=30$ packets.

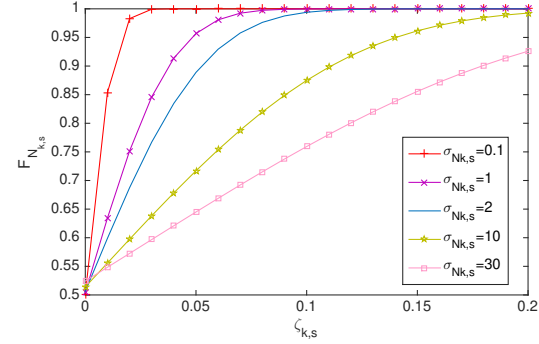

Fig. 11. CDF of number of transmitted packets versus delay margin for $\bar{N}_{k, s}=30$.

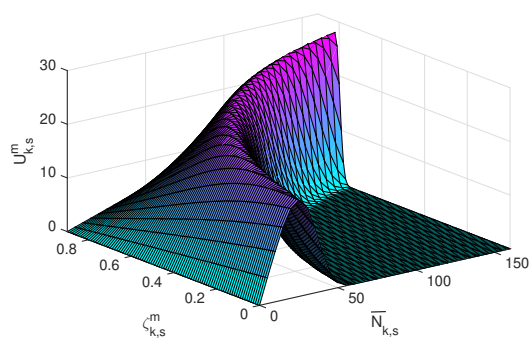

Fig. 14. Utilization of slice resources versus mean frame size and delay budget margin parameter for variation $\sigma_{k, s}=120$ and $N_{0}=30$ packets.
[7] K. Makhijani, K. Smith, J. Grant, A. Galis, and X. Defoy, "ETSI GR NGP 011 V1.1.1 next generation protocols (NGP) E2E network slicing reference framework and information model," https://www.etsi.org/deliver/etsi_ gr/NGP/001_099/011/01.01.01_60/gr_NGP011v010101p.pdf, September 2018.

[8] A. Galis and K. Makhijani, "Network slicing tutorial," in IEEE NetSoft 2018, Montreal 29th, June 2018.

[9] I. Afolabi, A. Ksentini, M. Bagaa, T. Taleb, M. Corici, and A. Nakao, "Towards 5G network slicing over multiple-domains," IEICE Transactions on Communications, pp. Vol. E100B, No. 11, 01.11.2017, p. 19922006, 2017.

[10] K. B. Letaief, W. Chen, Y. Shi, J. Zhang, and Y. A. Zhang, "The roadmap to 6G: AI empowered wireless networks," IEEE Communications Magazine, vol. 57, no. 8, pp. 84-90, 2019.

[11] X. Jiang, H. Shokri-Ghadikolaei, G. Fodor, E. Modiano, Z. Pang, M. Zorzi, and C. Fischione, "Low-latency networking: Where latency lurks and how to tame it," Proceedings of the IEEE, vol. 107, no. 2, pp. 280-306, Feb 2019.

[12] “3GPP TS 22.261 version 15.6 .0 Release 15 5G; service requirements for next generation new services and markets," https://www.etsi.org/deliver/etsi_ ts/122200_122299/122261/15.06.00_60/ts_122261v150600p.pdf, October 2018.

[13] M. Weiner, M. Jorgovanovic, A. Sahai, and B. Nikolie, "Design of a low-latency, high-reliability wireless communication system for control applications," in 2014 IEEE International Conference on Communications (ICC), June 2014, pp. 3829-3835.

[14] K. Yamamoto, F. Ichihara, K. Hasegawa, M. Tukuda, and I. Omura, "60 ghz wireless signal transmitting gate driver for igbt," in 2015 IEEE 27th International Symposium on Power Semiconductor Devices IC's (ISPSD), May 2015, pp. 133-136.

[15] A. Nasrallah, A. S. Thyagaturu, Z. Alharbi, C. Wang, X. Shao, M. Reisslein, and H. ElBakoury, "Ultra-low latency (ull) networks: The ieee tsn and ietf detnet standards and related $5 \mathrm{~g}$ ull research," IEEE Communications Surveys Tutorials, vol. 21, no. 1, pp. 88-145, Firstquarter 2019.

[16] D. Kreutz, F. M. Ramos, P. E. Verissimo, C. E. Rothenberg, S. Azodolmolky, and S. Uhlig, "Software-defined networking: A comprehensive survey," Proceedings of the IEEE, vol. 103, no. 1, pp. 14-76, 2015.

[17] F. Hu, Q. Hao, and K. Bao, "A survey on software-defined network and
OpenFlow: From concept to implementation," IEEE Communications Surveys \& Tutorials, vol. 16, no. 4, pp. 2181-2206, 2014.

[18] M. Yang, Y. Li, D. Jin, L. Zeng, X. Wu, and A. V. Vasilakos, "Softwaredefined and virtualized future mobile and wireless networks: A survey," Mobile Networks and Applications, vol. 20, no. 1, pp. 4-18, 2015.

[19] J. Qadir, N. Ahmed, and N. Ahad, "Building programmable wireless networks: an architectural survey," EURASIP Journal on Wireless Communications and Networking, vol. 2014, no. 1, p. 172, 2014.

[20] W. Guan, X. Wen, L. Wang, Z. Lu, and Y. Shen, "A service-oriented deployment policy of end-to-end network slicing based on complex network theory," IEEE Access, vol. 6, pp. 19691-19701, 2018.

[21] L. Wang, Z. Lu, X. Wen, R. Knopp, and R. Gupta, "Joint optimization of service function chaining and resource allocation in network function virtualization," IEEE Access, vol. 4, pp. 8084-8094, 2016.

[22] M. Leconte, G. S. Paschos, P. Mertikopoulosy, and U. Kozatz, "A resource allocation framework for network slicing," in IEEE International Conference on Computer Communications, April 2018, pp. 1-19.

[23] R. Kumar, M. Hasan, S. Padhyy, L. P. K. Evchenkoy, S. Mohany, and R. B. Bobbax, "End-to-end network delay guarantees for realtime systems using SDN," Real-Time Systems Symposium (RTSS), IEEE, 2017.

[24] B. Lu and Y. Zhang, "A mapping algorithm for low-latency network slices," in 2017 IEEE International Conference on Information and Automation (ICIA), July 2017, pp. 1156-1161.

[25] C. Sun, C. She, and C. Yang, "Energy-efficient resource allocation for ultra-reliable and low-latency communications," in IEEE Global Communications Conference (Globecom), 2017, pp. 1-6.

[26] C. She and C. Yang, "Ensuring the quality-of-service of tactile Internet," in 83rd IEEE Vehicular Technology Conference (VTC-Spring), 2016, pp. $1-5$.

[27] G. M. S. Rahman, M. Peng, and K. Zhang, "Radio resource allocation for achieving ultra-low latency in fog radio access networks," IEEE Access, vol. PP, no. 99, pp. 1-1, 2018.

[28] A. Fendt, S. Lohmuller, L. C. Schmelz, and B. Bauer, "A network slice resource allocation and optimization model for end-to-end mobile networks," in 2018 IEEE $5 G$ World Forum (5GWF), July 2018, pp. 262267.

[29] M. H. Hajiesmaili, M. S. Talebi, and A. Khonsari, "Multi-period network rate allocation with end-to-end delay constraints," IEEE Transactions on Control of Network Systems, vol. PP, no. 99, pp. 1-1, 2017. 
[30] J. Kleinberg and E. Tardos, Algorithm Design. Pearson Education, 2006.

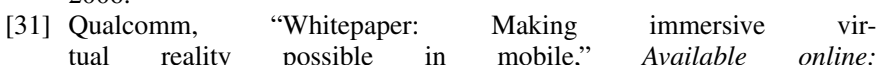
https://www.qualcomm.com/media/documents/files/whitepaper-makingimmersive-virtual-reality-possible-in-mobile.pdf, 2017.

[32] E. Bastug, M. Bennis, M. Medard, and M. Debbah, "Toward interconnected virtual reality: Opportunities, challenges, and enablers," IEEE Communications Magazine, vol. 55, no. 6, pp. 110-117, 2017.

[33] S. Kasiviswanathan, S. Eidenbenz, and G. Yan, "Geography-based structural analysis of the Internet," in https://pdfs.semanticscholar.org /cd7c/e2525ef56f36949ba75761587e211b63785a.pdf, 2010.

[34] A. Fei, G. Pei, and L. Liu, Roy Zhang, "Measurements on delay and hop-count of the Internet." in http://irl.cs.ucla.edu/papers/internetmeasurement.ps.gz, 1998.

[35] D. Watts and S. Strogatz, "Collective dynamics of 'small-world' networks," Nature, no. 393, pp. 440-442, 1998.

[36] P. Spira and A. Pan, "On finding and updating spanning trees and shortest paths," SIAM J. Computing, vol. 4, no. 3, pp. 375-380, 1975.

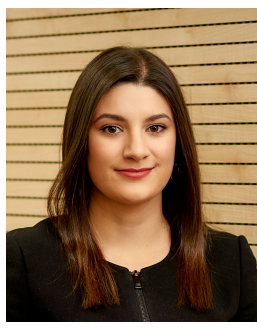

Ivana Kovacevic received the bachelor's degree in electronics and telecommunication engineering from the University of Belgrade, Serbia, in 2012 and the M.Sc. degree in communications engineering from the University of Oulu, Finland, in 2015, where she is currently pursuing the Ph.D. degree in wireless networks. In 2015, she joined the Centre for Wireless Communications, University of Oulu. Her research interest is in the area of network slicing, low latency communications, radio resource management, edge computing, spectrum sharing, network optimization theory, cognitive networks and game theory.

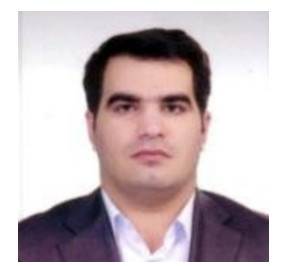

Alireza Shams Shafigh received the M.Sc. degree in computer engineering from the Iran University of Science and Technology, Iran, in 2006, and the $\mathrm{Ph} . \mathrm{D}$. degree from the University of Oulu, Finland, in 2018. He was a Faculty member of Islamic Azad University, Damavand Branch, from 2006 to 2012. He joined the Centre for Wireless Communications, University of Oulu, in 2013. His research interests are in the areas of user-centric networks, the Internet of Things, dynamic network slicing and software defined networks, end-to-end ultra high reliability, low latency and high security, and blockchain.

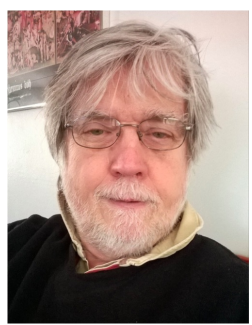

Savo Glisic (M'90-SM'94) is with University of Oulu, Finland, and INS Institute for Networking Sciences/ Globalcomm Oy. He was Visiting Scientist at Cranfield Institute of Technology, Cranfield, U.K. (1976-1977) and University of California, San Diego (1986-1987). He has been active in the field wireless communications for 30 years and has published a number of papers and books. The latest incoming book "Advanced Wireless Networks: Artificial Intelligence and Quantum Computing Enabling Communications, 4e, John Wiley and Sons, 2021" cover the enabling technologies for the definition, design and analysis of incoming $6 \mathrm{G} / 7 \mathrm{G}$ systems. His research interest is in the area of network optimization theory, artificial intelligence, block chain technology, cloud/edge/fog computing, networks information theory, network sciences, quantum channel information theory and quantum computing enabled communications. Dr. Glisic has served as the Technical Program Chairman of the third IEEE ISSSTA'94, the eighth IEEE PIMRC'97, and IEEE ICC'01. He was Director of IEEE ComSoc MD programs.

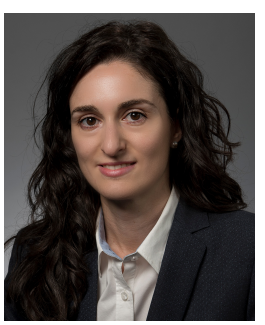

Beatriz Lorenzo received the Ph.D. degree from the University of Oulu, Finland, in 2012, and the M.Sc. degree in telecommunication engineering from the University of Vigo, Spain, in 2008. She is an assistant professor in the Department of Electrical and Computer Engineering at the University of Massachusetts, Amherst, and she is also a distinguished researcher at the University of Vigo, Spain. Her research interests include wireless networks, network architectures and protocol design, mobile computing, optimization, and network economics. She received the Fulbright Visiting Scholar Fellowship, University of Florida, from 2016 to 2017.

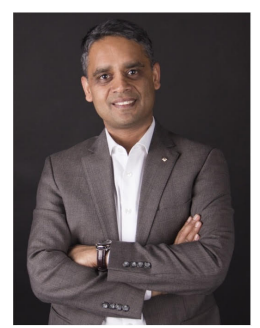

Ekram Hossain (F'15) is a Professor and the Associate Head (Graduate Studies) in the Department of Electrical and Computer Engineering at University of Manitoba, Canada. He is a Member (Class of 2016) of the College of the Royal Society of Canada. Also, he is a Fellow of the Canadian Academy of Engineering. Dr. Hossain's current research interests include design, analysis, and optimization beyond $5 \mathrm{G}$ cellular wireless networks. He was elevated to an IEEE Fellow "for contributions to spectrum management and resource allocation in cognitive and cellular radio networks". He was listed as a Clarivate Analytics Highly Cited Researcher in Computer Science in 2017, 2018, and 2019. Currently he serves as the Editor-in-Chief of IEEE Press and an Editor for the IEEE Transactions on Mobile Computing. Previously, he served as the Editor-inChief for the IEEE Communications Surveys and Tutorials (2012-2016). He is a Distinguished Lecturer of the IEEE Communications Society and the IEEE Vehicular Technology Society. Also, he is an elected member of the Board of Governors of the IEEE Communications Society for the term 2018-2020. 